\title{
The Narrative Potential of the British Birth Cohort Studies
}

\author{
Jane Elliott \\ Centre for Longitudinal Studies \\ Institute of Education \\ 20 Bedford Way \\ London WC1H OAL \\ J.Elliott@ioe.ac.uk \\ 02076126395 \\ Fax 02076126997
}

Jane Elliott is Reader in Research Methodology at the Institute of Education and

Research Director of the 1958 and 1970 British Birth Cohort Studies at the Centre for

Longitudinal Studies. Her research interests include gender, employment and family life and she has a longstanding methodological interest in narrative and combining qualitative and quantitative research methods. 


\begin{abstract}
This paper draws attention to the narrative potential of longitudinal studies such as the British Birth Cohort Studies (BBCS), and explores the possibility of creating narrative case histories and conducting narrative analysis based on information available from the studies. The BBCS have historically adopted a quantitative research design and used structured interviews and questionnaires to collect data from large samples of individuals born in specific years. However, the longitudinal nature of these studies means that they follow the same sample of individuals from birth through childhood into adult life, and this leads to the creation of data that can be understood as a quantitative auto/biography.
\end{abstract}

\title{
Key words: narrative; longitudinal research; cohort studies; mixed- methods research
}

\section{Introduction}

Compared with the explosion of interest over the past twenty years in the prevalence of narratives within qualitative data, there has been far less attention on the possibility of constructing narratives with quantitative data collected using survey methods. However, the growing interest in longitudinal approaches to research means that quantitative analysis increasingly has narrative, or at least chronological, features. Indeed, the very rich longitudinal data now available facilitate innovative approaches to analysis, and allows for qualitative and quantitative techniques to be combined in new ways. 
In particular, the focus is on the British Birth Cohort Studies (BBCS), which have collected a continuous record of data on samples of individuals from birth

through childhood and well into adult life. The first section of the paper provides a brief introduction to the type of data collected in large-scale longitudinal studies and an outline of the main ways in which statistical models and numerical summaries of individual lives may be understood and presented in more narrative terms. The second section focuses more specifically on the potential for doing narrative analysis using the British Birth Cohort Studies and highlights new opportunities for analyzing the more qualitative textual material that is embedded within each study.

\section{Quantitative longitudinal data: narrative potential and narrative limitations}

Longitudinal data can broadly be understood as any information that tells us about what has happened to a set of research cases over a series of time points. The majority of longitudinal research focuses on individuals as the unit of analysis and usually records change at an individual or micro-level (Ruspini, 2002). Longitudinal data is either collected prospectively or retrospectively.

Prospective research designs involve repeatedly returning to the same sample or panel and asking people about their current circumstances (for example, marital status, the domestic division of labour, employment, job satisfaction, housing, income, physical health and mental health). This results in 
a detailed record being compiled of how individual circumstances and experiences change over time. Retrospective methods involve asking people to recall and record information about the past. This is very common in large-scale surveys and has become an established method for obtaining information about the dates of key events in people's biographies. For example, in large-scale longitudinal studies such as the British Household Panel Survey, the British Birth Cohort Studies and the National Longitudinal Studies of Youth in the United States, quantitative retrospective life history information is typically collected on the dates of cohabitations, marriages, separations and divorces, the births of any children, dates of moving house and of job changes or promotions. It was my experience of working with these retrospective life histories that first made me aware of the parallels and differences between this type of very structured data and the stories that people tell about their lives.

Statistical analysis may initially appear to be a completely different enterprise from constructing a narrative, partly because it is based on manipulating numeric summaries or measures rather than textual evidence. However, I would argue both that statistical analysis frequently has an implicit narrative form and that quantitative data can be used in innovative ways to produce narratives about individuals. There are perhaps four main ways in which the analysis of quantitative, in particular longitudinal, data can be understood to have a narrative character. 
First, any chronological dimension to quantitative data encourages an analysis of how a combination of variables produces a specific outcome over time for a sample of individuals. While a narrative often has a stated resolution, the aim of a quantitative analysis is usually to identify the main factors influencing a particular outcome, often referred to as the dependent variable. In a sense, therefore, in a statistical model the outcome of the dependent variable performs the same function as the resolution in a narrative. It is in relation to the dependent variable that other factors are judged to be either significant or not in explaining the specific outcome. Similarly, just as an effective narrative involves the selection of important events, rather than the inclusion of every detail, statistical modelling enables the researcher to identify the most salient factors, or those variables that are most strongly associated with later outcomes.

Second, in the same way that a narrative has an orientation that situates it in time and place, a statistical model, or a set of descriptive statistics, is based on the analysis of a particular sample of data that is representative of a population which is historically and geographically situated. As discussed below regarding the BBCS, by comparing the results of statistical analyses carried out on samples of individuals born into different generations, researchers can develop analytical narratives about social change. These might be thought of as meta-narratives, in that they are not about specific individuals but change at the aggregate level. For example, a number of researchers have used data from the decennial censuses in Britain to examine how occupational segregation by sex has changed over 
time (Hakim, 1994; Blackwell and Guinea-Martin, 2005). This has indicated the diminishing tendency of men and women to do different kinds of paid work, and to be concentrated into different types of occupations, over the twentieth century and particularly over the last three decades.

Third, although conventional approaches to the analysis of quantitative data tend to obscure the individual by presenting results at an aggregate level (Abbott, 1992), the form of the data may make it possible to reconstruct descriptive profiles of the members of a sample. These profiles will come closest to having a narrative form where the quantitative data is very detailed and longitudinal. Whether data has been collected prospectively or retrospectively, the chronological dimension of longitudinal data facilitates the construction of biographies that document change over time for specific individuals. This is most effective where data has been collected prospectively over a number of years, because it is then possible to combine data collected several years apart, in order to provide a more holistic account of an individual's life trajectory.

These individual case studies can be used to illustrate the results of more conventional quantitative analysis. However, they can also be used to stimulate hypotheses about the factors that work in combination to produce particular outcomes for individuals and in some cases as a precursor to more conventional statistical analysis. Here, the rationale for recasting longitudinal survey data in the form of a narrative is that the human mind is better suited to process the 
information embedded in a coherent story than to grasp long lists of variables about a single life (Singer et al, 1998: 19).

However, what is missing from these reconstructed individual stories is the individual's evaluation of the events that are recorded. To this extent, each individual life history might be thought of as closer to a chronicle rather than fully realizing a narrative form (White, 1987). There is a danger that, unlike a fully formed narrative, a life history based on quantitative data 'does not so much conclude as simply terminate; typically it lacks closure, that summing up of the meaning of a chain of events with which it deals that we normally expect from the well made story' (White, 1987: 16). ${ }^{1}$

Fourth, more innovative narrative approaches to analysis become possible as an increasing number of large-scale quantitative studies include the collection of qualitative textual material. For example, in addition to responding to fixed choice questions, respondents are occasionally asked more open-ended questions permitting slightly more heterogeneous responses. Thus the British Household Panel Survey (BHPS) predominantly collects quantitative information, although each year respondents are also asked about any significant events that have occurred in the year since they were last interviewed. Additionally, in 2001, members of the panel were also asked to describe the main advantages and disadvantages of being their age. This textual material, together with a detailed quantitative record about each individual, increasingly makes it possible to 
produce case studies that draw on a combination of qualitative and quantitative material to produce understandings of individual lives within a broader social context.

Despite the apparent contrast between quantitative analysis and more narrative approaches to inquiry, which often start from individual accounts and detailed textual material, there are therefore a number of ways in which quantitative data can be used by researchers to construct narratives. As I have argued elsewhere (Elliott, 2005), recognition of the narrative character of much quantitative research could usefully lead to greater reflexivity among quantitative researchers and help productive methodological discussions about how quantitative and qualitative approaches to analysis may be combined, even if not fully integrated. In the following section, I discuss the British Birth Cohort Studies to suggest the types of narratives which can be crafted from quantitative data and argue that, of all the quantitative resources currently available, it is cohort studies which have the most potential for narrative research.

\section{The British Birth Cohort Studies as a resource for narrative inquiry}

Britain is unique in having three national birth cohort studies that have followed individuals, born in a specific week of the year, through childhood and adolescence and into adult life. The first began as a survey of maternity services in 1946 and was followed by a second study twelve years later in 1958 and a 
third in 1970. It is beyond the scope of this paper to provide a summary of the wide body of research based on these four studies, but for brief descriptive accounts and some of the main results, see Wadsworth 1991; Ferri, Bynner and Wadsworth 2003; Power and Elliott 2006; Elliott and Shepherd 2006. The Millennium Cohort Study (MCS) represents the fourth British Birth Cohort Study and has already collected detailed information about children and their parents at age 9 months, 3 years, 5 years, and 7 years; and for early results see Dex and Joshi (2005). In comparison with the earlier cohorts, the Millennium Cohort study is still in its infancy and cannot yet provide insights into individual trajectories from childhood and into adult life. The focus here is therefore on the earlier cohorts and particularly the 1958 and 1970 studies.

The majority of information collected in all four of the cohort studies is quantitative, and the researchers who use these resources almost exclusively adopt a quantitative analytic approach, for example, estimating multivariate and longitudinal models in order to understand more about the ways that different variables are associated with each other. However, the detailed and longitudinal nature of the studies, and the fact that cohort members are explicitly located in a specific historical context, means that the studies have a number of narrative properties. I shall now explore the narrative potential of these studies and also underline the limitations of quantitative data for producing narratives that allow for the reflexivity of the individual to be appreciated and the perspectives of the respondents themselves to be heard. In the final section I shall highlight new 
opportunities for analysing the more qualitative information embedded within each study and argue there is scope for innovative work which weaves together the different types of evidence to produce new narrative forms.

\section{Narratives of social change}

Longitudinal research based on data from a group of individuals all born at the same time, such as the British cohort studies, has considerable potential for allowing researchers to frame the results of their analyses within a specific historical and cultural context. In particular, when cross-cohort comparisons are made, there is potential for examining how changes in society might shape the way that individuals experience particular events or difficulties in their lives and, in addition, might reduce or increase the impact of those events on individual outcomes. For example, it has been suggested that the negative impact of parental divorce on children may be reduced as divorce becomes more common. Research by Margaret Ely et al, which examines the association between having divorced parents and educational outcomes for three cohorts of British children born in 1946, 1958 and 1970, was able directly to test this reduced effect hypothesis (Ely et al, 1999) and demonstrates there is little evidence that the impact of divorce has diminished for more recent cohorts of children.

Not all researchers using data from the cohort studies pay close attention to the historical specificity of the data. However, a growing number of 
publications have explicitly compared results of analysis of data from two or more cohorts and used this to construct an account of social change (Ferri et al, 2003; Galindo-Rueda and Vignoles, 2003; Blanden and Gibbons, 2006). What is significant here is that, although the authors are applying a purely quantitative analytic approach, they can be understood as using the cohort data to produce narratives at two different levels. First, the statistical models they construct are designed to explore how circumstances, characteristics and experiences in early life impact on outcomes in adult life. These models can be said to rely on an implicit narrative or auto/biographical structure, in which the reader expects events to unfold over time and culminate in some kind of resolution. Second, research which compares information from two or more cohorts is also constructing a meta-narrative about social change. For example, both GalindoRueda and Vignoles (2003) and Blanden and Gibbons (2006) suggest that British society is becoming less meritocratic and that opportunities and outcomes in people's adult life are becoming more dependent on the economic aspects of their family background.

\section{Constructing individual case histories}

The type of statistical modelling used in the quantitative research described above could therefore be said to result in a type of social or group narrative, because factors measured in early life are identified as appearing to impact on later outcomes. For example, at the aggregate level having divorced 
parents or being economically disadvantaged during childhood can be shown to have a significant impact on the probability of gaining qualifications or having a well-paid job in adult life. It is the prospective longitudinal nature of the data in the cohort studies that facilitate these kinds of analyses, which have at their heart an implicit narrative form with a chain of events leading to a specific outcome or resolution. However, lay audiences, and those wanting to use research to inform policy, can find multivariate statistical analyses rather dry and impenetrable. There is also a sense in which statistical (i.e. quantitative) descriptions of groups of people do not do justice to the vibrant and unique individuals who are the subjects of the research (Farran, 1990; Pugh, 1990).

For these reasons, some sociologists have experimented with providing case studies of individuals to illustrate the results of their research. For example, Bynner et al, (1997) provide descriptions of trajectories followed by a number of typical individuals from the 1958 and 1970 British cohort studies to complement their statistical descriptions of the longitudinal data. A slightly different approach has been taken by Joshi and Davies (1996), who have simulated earnings over a life time for a small number of fictional cases, to characterize the most common employment trajectories of different groups of women.

The British Birth Cohort Studies provide ideal sets of data with which to compile detailed individual biographies, which can either be used to illustrate the results of statistical analysis or to generate new hypotheses. For example, the 
1958 study includes over 15,000 variables (i.e. pieces of information) about each cohort member. During childhood, much of this data was collected from parents, teachers and medical professionals, while in adult life the majority has been collected from the cohort members themselves. The multipurpose nature of the study means that data are available on growth and development; physical and psychological health and well-being; educational experiences and attainment; cohabiting relationships and family formation; housing; employment; social participation; values and attitudes. It is therefore possible to use these rich resources to create individual biographies that focus on a particular theme or which document the inter-relation between the different domains in an individual's life. These individual biographies can in turn be contextualized using descriptive quantitative information from the whole cohort. For example, we not only have information about whether a specific cohort member lived in local authority housing at age eleven, but we can also understand this in the context of knowing that $42 \%$ of the cohort lived in local authority housing at the time.

To some extent, therefore, interpretations of the meaning of specific experiences for the individual are informed by the data on the whole cohort. This provides a quantitative description of the contemporary social context, an orientation for each individual narrative. However, we should be clear that, by using quantitative data in this way, it is the researcher who remains the narrator of each biography. The individuals whose lives are being presented do not have a voice. The meanings that they might attach to specific experiences or 
circumstances during their lives are obscured from view. It is the researcher who selects what appears to be most interesting or significant from the wealth of material that is available and who crafts the life stories which can never fully represent the reflexive individuals, who have their own stories to tell. However, as discussed in the next section, there are places within the British Birth Cohort Studies where the cohort members themselves do have a voice, or at least are given an opportunity to provide more qualitative or textual data that could allow for a different kind of narrative to be constructed.

\section{Qualitative textual data}

Although the majority of information collected in the British Birth Cohort Studies is quantitative, there is also some qualitative textual material available as a resource for qualitative or mixed methods analysis. In addition, the fact that these textual data are part of a longitudinal archive opens up opportunities for a more narrative approach to analysis, even if the individual texts are not narratives in themselves. This final part of the paper will provide a brief summary of these textual resources, and also argue that their value can most fully be realised when they are combined with the detailed longitudinal quantitative data from the studies.

Perhaps the richest source of qualitative data within the cohort studies is a set of essays written by members of the 1958 cohort when they were eleven in 
1969. Specifically, the children were asked to write an essay about what they thought their life would be like at age 25 .

A total of 13,669 children completed this essay, and a sub-sample of approximately 500 of these essays (average word length 187 words) have now been transcribed and coded for their main themes (Elliott and Morrow, 2007). This sub-sample is in process of being archived at the UK Data Archive in Essex, and will be available for other researchers to use. An example of a relatively short essay written by a girl aged eleven is provided below:

Serial number: $435040 R$

If I was 25 I would start studying to be a Wren. When I had studied for as long as I should, I would start as a secretary and perhaps the captain of a ship would invite me onto his ship to go for a voyage to another country or a small island. I would learn a few languages perhaps and make friends with people from other countries. During the holidays I would go and visit my family. Perhaps meet a jolly sailor and have a family of my own.

Words: 90

This essay has aspects of a narrative form in its clear progression from 'I would start studying to be a WREN' to the happy ever after of meeting a jolly sailor and having a family, while some other essays were more descriptive and 
lacked a clear temporal dimension. But what makes all these essays amenable to narrative analysis is that they are embedded within the record of the children's circumstances when they wrote the essays at age 11 and the detailed information about their subsequent lives recorded at ages 16, 23, 33, 42 and 46 years.

Qualitative textual data has also been collected in the other two cohort studies. When members of the 1970 cohort were 16 , they were asked to complete a number of questionnaires about their aspirations, leisure activities, friendships and health related behaviour and also to provide brief written responses to a number of open-ended questions. These covered topics including the individual's aspirations, understanding of AIDS, descriptions of football violence, and ideas about the causes and remedies for this violence. They were all asked whether they had Received any unwanted sexual attention. Responses to these questions have been transcribed, but to date have not been analysed in detail. Some descriptions of unwanted sexual attention include:

'A boy at a disco tried to grab my bum and undo my skirt, I poured my drink over him'

'Myself and some friends were flashed at whilst walking home from a netball match' 
Here a total of 852 sixteen year olds ( $14 \%$ of those who returned the questionnaire) provided a brief written summary of what happened to them. There was a clear gender difference in responses, so that 164 (6\%) boys and $688(20 \%)$ girls) stated that they had received unwanted sexual attention. Even though the responses are not detailed, the number of individuals who answered this open-ended question means there is a great deal of qualitative material that could be analysed, either in conjunction with the quantitative data or as a resource in its own right - for example, concerning discourses used by teenagers around the experience of unwanted sexual attention.

The 1946 cohort study also includes some qualitative material still relatively under-utilised to date. In adult life, cohort members were asked to make comments, at age 26 , comparing how they felt they were getting on in the world compared with their parents and contemporaries, and at age 36 were asked to list the things they wish they had done differently in education and family life. As part of a focused sub-study on women's health, which involved sending a postal questionnaire to women in the cohort study between age 47 and age 54, women were encouraged to include free text comments about their experiences of the menopause, providing a more qualitative account of women's experiences of the change of life (Ballard et al, 2001).

In 2006, a programme of scanning the substantial accumulation of paper and fiche records generated over the years by the 1958 and 1970 birth cohort 
studies, was carried out, funded by the UK's Economic and Social Research Council. Consequently much of the qualitative material associated with the cohort studies is now more readily accessible to the research community.

\section{Cohort members' narratives}

There will be further opportunities in the future for linking narrative material with quantitative data from the cohort studies. In particular, a qualitative substudy of approximately 180 cohort members has been planned as part of the data collection from members of the 1958 cohort at age 50 in 2008 . Following the structured quantitative interview, a sub-sample of cohort members will be invited to take part in an in-depth biographical interview. Cohort members will be asked about their current social participation, including contact with family and friends, active membership of groups and voluntary organisations. They will also be asked to provide their life story focusing on perceptions of the major influences on their life and any major turning points. Building on the work of Cote and Levene (2002) and Preston (2004), the aim is to discover the identity strategies used by cohort members and how these intersect with, and are shaped by, individual biographies.

The transcribed interviews will be archived so that they are available for secondary analysis, either as qualitative data or in tandem with the rich quantitative data that already exists about members of the cohort. As highlighted 
above, the detailed quantitative data available from the cohort studies already allows for the construction of biographies about individuals. However, these lack

the perspective of the cohort members themselves. The data currently available, however detailed, can never provide a complete record of an individual's life, and the information collected over the years is necessarily a product of the research agendas of the social scientists responsible for executing the study. The collection of qualitative life histories from cohort members will therefore add a whole new dimension to the research.

\section{Conclusions}

This paper has explored the narrative properties of the British Cohort Studies, focusing on the 1958 and 1970 birth cohort studies managed at the Centre for Longitudinal Studies. Although the majority of this data is quantitative, I have outlined some of the qualitative textual data available from the studies, or which will be in the near future. The detailed information collected on cohort members over the years, coupled with the potential for understanding analysis of individual trajectories within specific historical and social contexts, goes some way to allowing researchers to construct narrative biographies or case histories of individuals within these studies. However, members of the cohort studies have, to date, been given limited, if any, opportunity to tell their own life stories. The narratives that can be constructed from existing quantitative data are therefore likely to reflect the preoccupations and interests of the researchers who 
have contributed to the content and design of the studies, rather than the perspectives of the people whose lives have been charted in detail over the past five decades. But there is considerable potential here for researchers with an interest in individual lives and the construction of biographical case histories to use the data collected in these unique studies in innovative ways. 


\section{References}

Abbott, A. (1992) 'What do cases do?', in C. Ragin and H. S. Becker (eds) What is a case? (pp. 53-82). Cambridge: Cambridge University Press.

Ballard, K., Kuh, D. and Wadsworth, M. E. J. (2001) 'The role of the menopause in women's experiences of the 'change of life", Sociology of Health and IIIness 23: 397-424.

Blackwell, L. and Guinea-Martin, D. (2005) 'Occupational segregation by sex and ethnicity in England and Wales, 1991 to 2001', Labour Market Trends 113(12): 501-516.

Blanden, J. and Gibbons, S. (2006) The persistence of poverty across generations: A view from two British cohorts. Bristol: Joseph Rowntree Foundation.

Bynner, J. and Fogelman, K. (1993) 'Making the Grade: education and training experiences', in E. Ferri (ed.) Life at 33: The fifth follow-up of the National Child Development Study (pp. 36-59). London: National Children's Bureau.

Bynner, J., Ferri, E. and Shepherd, P. (1997) Twenty-something in the 1990s: getting on, getting by, getting nowhere. Aldershot: Ashgate.

Côté, J. and Levene, C. (2002) Identity formation, agency and culture. New Jersey: Lawrence Erlbaum.

Dex, S. and Joshi, H. (2005) Children of the 21st Century: From birth to nine months. Bristol: The Policy Press.

Elliott, J. (2005) Using Narrative in Social Research: Qualitative and Quantitative Approaches. London: Sage.

Elliott, J. and Shepherd, P. (2006) 'Cohort Profile: 1970 British Birth Cohort (BCS70)', International Journal of Epidemiology 35: 836-843.

Elliott, J. and Morrow, V. (2007) 'Imagining the Future: preliminary analysis of NCDS essays written by children at age 11'. London: Centre for Longitudinal Studies, Institute of Education.

Ely, M., Richards, M. P. M., Wadsworth, M. E. J. and Elliott, B. J. (1999) 'Secular Changes in the Association of Parental Divorce and Children's Educational Attainment - Evidence from Three British Birth Cohorts', Journal of Social Policy 28(3): 437-455. 
Farran, D. (1990) 'Seeking Susan: producing statistical information on young people's leisure', in L. Stanley (ed.) Feminist praxis (pp. 91-102). London:

Routledge.

Ferri, E., Bynner, J. and Wadsworth, M. E. J. (2003) Changing Britain, Changing Lives: Three Generations at the Turn of the Century. London: Institute of Education.

Galindo-Rueda, F. and Vignoles, A. (2003) 'Class Ridden or Meritocratic? An Economic Analysis of Recent Changes in Britian'. London: Centre for the Economics of Education.

Hakim, C. (1994) 'A Century of Change in Occupational Segregation', Journal of Historical Sociology 7: 435-454.

Joshi, H., Davies, H. and Land, H. (1996) The tale of Mrs Typical. London: Family Policy Studies Centre.

Power, C. and Elliott, J. (2006) 'Cohort Profile: 1958 British birth cohort (National Child Development Study)', International Journal of Epidemiology 35: 34-41.

Preston, J. (2004) 'Identity, learning and engagement: a qualitative enquiry using NCDS'. London: Centre for the Wider Benefits of Learning, Institute of Education.

Pugh, A. (1990) 'My statistics and feminism - a true story', in L. Stanley (ed.) Feminist praxis (pp. 103-112). London: Routledge.

Ruspini, E. (2002) Introduction to Longitudinal Research. London: Routledge.

Singer, B., Ryff, C. D., Carr, D. and Magee, W. J. (1998) 'Linking life histories and mental health: A person-centered strategy', Sociological Methodology 28: 151.

Wadsworth, M. E. J. (1991) The Imprint of Time: Childhood, history and adult life. Oxford: Oxford University Press.

White, H. (1987) The Content of the Form. Baltimore: John Hopkins. 
' White is not discussing quantitative data here but comparing historical annals, chronicles and narratives. However, it is striking how closely his description of a historical chronicle matches the characteristics of a quantitative event history. 